\title{
BEAM TRAJECTORY JITTER IN THE SLC LINAC*
}

\author{
Chris Adolphsen and Tim Slaton \\ Stanford Linear Accelerator Center, Stanford University, Stanford, CA 94309 USA
}

We present model-independent measurements of the vertical trajectory jitter of the positron beam in the Stanford Linear Collider (SLC) linac and discuss the results of studies aimed at isolating its source.

\section{INTRODUCTION}

In March 1993, the SLC began flat beam operation which reduced the vertical emittance at the beginning of the linac by an order of magnitude to about $3 \times 10^{-6} \mathrm{~m}$-rad. Although this improved the average luminosity, it made the luminosity more sensitive to vertical beam jitter, that is, to the variations of the vertical orbits that are faster than the few second response time of the feedback loops that are used to stabilize the orbits. The rms of the vertical jitter, which had been observed to grow in size along the linac, now became a significant fraction of the vertical beam size. In this paper, we present some of the jitter measurements and discuss the results of studies aimed at isolating its source. In particular, we examine the positron jitter since the analysis of the electron jitter is complicated by its dependence on the positron motion via long-range wakefields. However, the electron results do not differ greatly.

\section{MEASUREMENTS}

To characterize the jitter, we wanted an empirical method since the linac beam transport is difficult to accurately model due to uncertainties in the linac energy profile and the strong transverse wakefield effects. Our solution was to measure the beam transport properties by inducing betatron oscillations near the injection point to the linac and recording the beam trajectory changes along the linac. Using these data, we characterized trajectory changes that were measured in different regions of the linac in terms of an equivalent injection jitter, and thus mapped how the jitter varied along the linac in common units. To make the measurements more relevant to SLC operation, we normalized the results to the beam size measured at injection. However, when interpreting the results, we also had to keep in mind the non-jitter emittance growth that occurred in the linac and in the subsequent transport of the beams to the collision point.

\footnotetext{
* Work supported by Department of Energy contract DEAC03-76F00515.
}

In the study of the positron vertical jitter, betatron oscillations were measured in five regions along the linac. The regions each span about two betatron oscillation wavelengths and are separated by about four wavelengths. Each region contained a series of 24 beam position monitors (BPMs) with an average resolution of about $10 \mu \mathrm{m}$ at the nominal beam intensity of $3.4 \times 10^{10}$ positrons per bunch. Prior to the measurements, data were taken to obtain the $R_{33}$ and $R_{34}$ TRANSPORT matrix elements from an upstream reference point to the BPMs in each of the regions (the $x-y$ coupling is negligible in the linac and so the other matrix elements were ignored). The reference point was chosen as the location of first beam size monitor (BSM) in the linac which is part of a set that is used to monitor beam emittance.

The $R_{33}$ and $R_{34}$ matrix elements for the positron beam were measured using two vertical steering dipole magnets (YCORs) in the injection line to the linac that are 90 degrees apart in betatron phase advance. The setting of each YCOR was varied in 11 steps while the linac BPMs were recorded with a five pulse average. The induced oscillations at the extreme YCOR settings were made much larger than the nominal beam jitter and BPM resolution so an accurate measurement could be made. The slopes of the BPM -vs- YCOR data were then fit for each BPM and the results used to compute the TRANSPORT elements. Because the YCORs are upstream of the BSM, this transformation can be done independently of the YCOR settings with only a knowledge of the distance of the BSM to its two neighboring BPMs. The resulting values for $R_{33}$ and R34 agree well with the linac optics model near the beginning of the linac but lag in phase and increase in amplitude further along the linac.

After measuring the coefficients, several sets of jitter data were taken during $120 \mathrm{~Hz}$ operation in which the BPM readings in the five regions were simultaneously recorded on 200 consecutive pulses. In each region, the mean orbit was subtracted from the BPM data and results were fit using the measured $R_{33}$ and $R_{34}$ matrix elements to obtain the orbit parameters $y$ and $y^{\prime}$ at the BSM. The orbit parameters were then normalized using typical values of the measured vertical beam size, $\sigma_{\mathrm{y}}=132 \mu \mathrm{m}$, and TWISS parameters, $\beta_{y}=13.5 \mathrm{~m}$ and $\alpha_{y}=-5.8$, at the BSM (the TWISS parameter calculation uses beam size data from the other BSMs in the set as well). Specifically, $\mathrm{Y} \equiv \mathrm{y} / \sigma_{\mathrm{y}}$ and $\mathrm{Y}^{\prime} \equiv\left(\alpha_{\mathrm{y}} \mathrm{y}+\beta_{\mathrm{y}} \mathrm{y}^{\prime}\right) / \sigma_{\mathrm{y}}$ were computed for each region of the linac from the data from each pulse. For jitter with a 
phase space distribution equal to that of the beam, $\mathrm{Y}$ and $\mathrm{Y}$ would each have an rms of unity and be uncorrelated.

Figure 1 shows examples of the distributions from a set of data that were taken with the linac feedback loops disabled. Here $Y^{r}$ - vs- $Y$ is plotted for the first and last region of the linac, which are denoted by the linac sector at the center of the region (note that the main linac is divided into 29 sectors: LIO2 to LI30). The measurement errors on the data points, which are not shown in the plots, are much smaller than the rms variation of the data. A comparison of the two plots clearly shows that only a small portion of the trajectory variation at the end of the linac can be attributed to injection jitter. The results for data taken with the linac feedback loops enabled are similar.
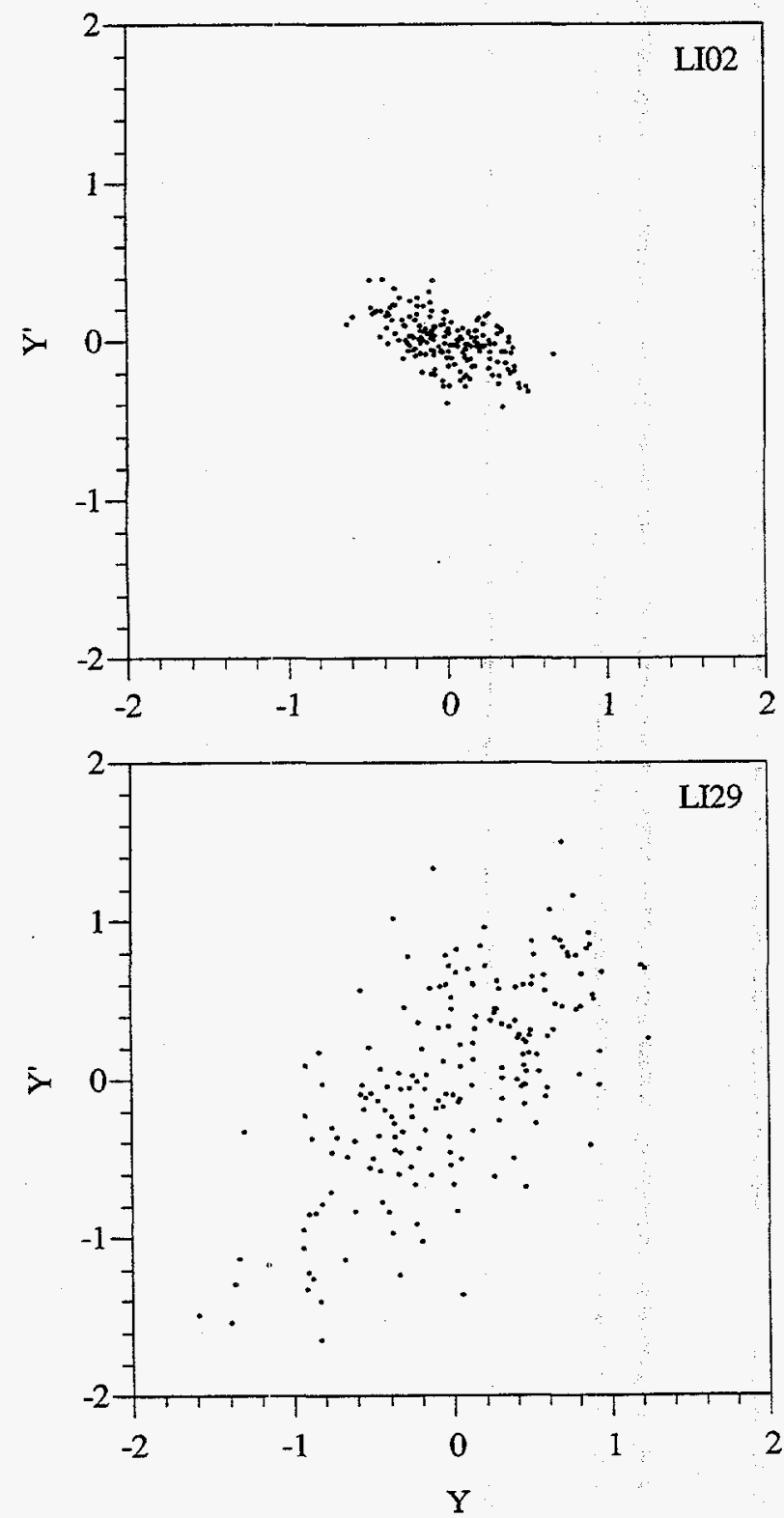

Figure 1: Phase space of vertical positron jitter measured in linac sectors LIO2 and LI29.
To quantify the jitter, we computed an rms of the rms variation of $Y$ and $Y^{\prime}$,

$$
\mathrm{Ts} \equiv \sqrt{\left\langle\mathrm{Y}^{2}\right\rangle+\left\langle\mathrm{Y}^{2}\right\rangle} / \sqrt{2}
$$

which is related to the time-averaged beam emittance growth in the presence of jitter, $\Delta \varepsilon / \varepsilon \approx \mathrm{Ts}^{2}$. The values of Ts for the feedback-disabled data are plotted in Figure 2 for the five linac regions. One sees that much of the positron vertical jitter growth occurred near the end of the linac. The LI 29 value of $60 \%$ would have contributed $36 \%$ to the time-averaged beam emittance in LI29 if no other emittance growth had occurred in the linac. However, the vertical emittance typically doubled in the linac, independent of the jitter, so the addition of the jitter had increased the time-averaged emittance by $18 \%$ in LI29. At the interaction point (IP), the jitter was about the same as in LI29 but the vertical emittance was typically a factor of three larger, so the jitter related emittance growth was only $6 \%$. The size of the vertical electron IP jitter was similar to, and essentially uncorrelated with the positron jitter, so the collision luminosity was reduced by about $4 \%$ due to vertical jitter (the horizontal jitter reduced it by an additional $3 \%$ in quadrature) [1].

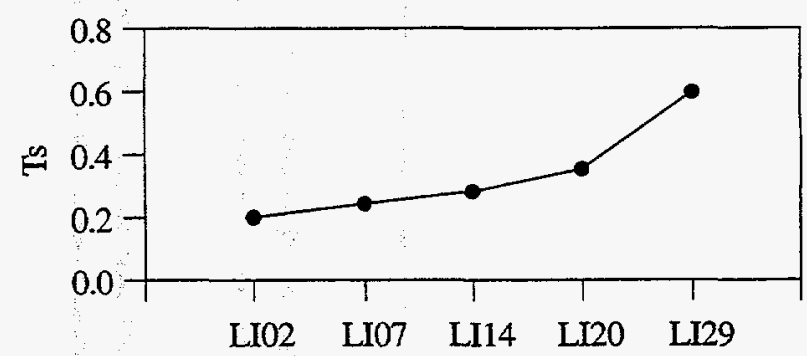

Figure 2: Ts values measured in five regions of the linac.

To study the temporal dependence of the jitter, Fourier transforms of the jitter data from the five regions were computed. The time dependence of the projections of the jitter along the major and minor axes of the phase space ellipses were analyzed in addition to $Y$ and $Y^{\prime}$. As an example, Figure 3 shows the power spectrum (PS) and integral of the power spectrum (IS) for the LI02 and LI29 jitter projected along the major axes of their phase space ellipses. Note that for this analysis the jitter data was normalized to have an rms of unity, so the integrated spectrum, which sums the power spectrum above the frequency plotted; is unity at $0 \mathrm{~Hz}$. The relative rms jitter due the Fourier components above a given frequency is therefore the square root of the IS value at that frequency. In general, the measured spectra are essentially white noise with a few prominent frequency components in some cases. However, these components seldom contribute more than $10 \%$ to the total rms. 


\section{DISCLAIMER}

This report was prepared as an account of work sponsored by an agency of the United States Government. Neither the United States Government nor any agency thereof, nor any of their employees, makes any warranty, express or implied, or assumes any legal liability or responsibility for the accuracy, completeness, or usefulness of any information, apparatus, product, or process disclosed, or represents that its use would not infringe privately owned rights. Reference herein to any specific commercial product, process, or service by trade name, trademark, manufacturer, or otherwise does not necessarily constitute or imply its endorsement, recommendation, or favoring by the United States Government or any agency thereof. The views and opinions of authors expressed herein do not necessarily state or reflect those of the United States Government or any agency thereof. 


\section{DISCLAIMER}

Portions of this document may be illegible in electronic image products. Images are produced from the best available original document. 

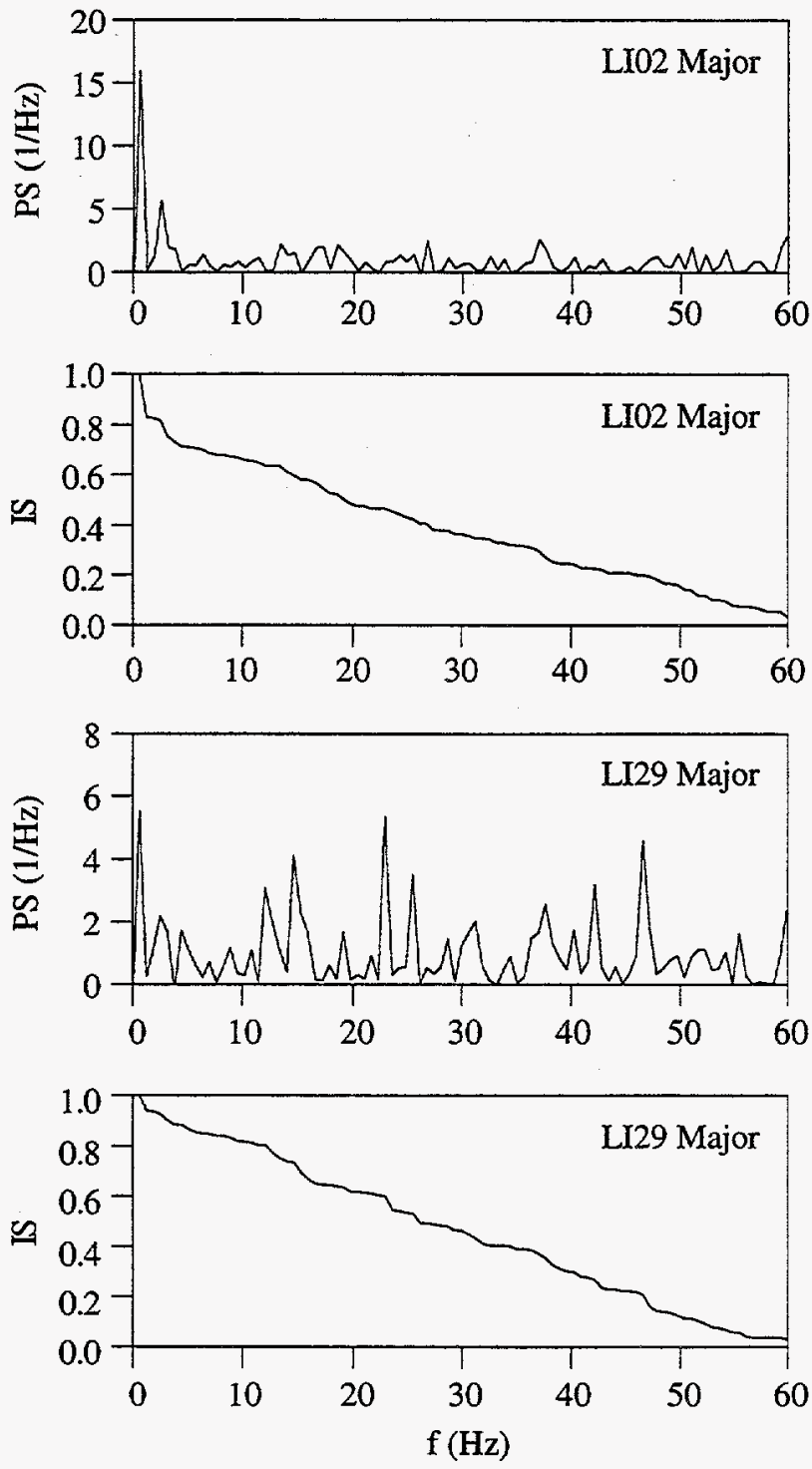

Figure 3: Normalized power spectrum (PS) and integral of the power spectrum (IS) for the LI02 and LI29 jitter projected along the major axes of their phase space ellipses.

\section{DISCUSSION}

Although the source of the jitter growth in the linac is not yet understood, the results from various studies have narrowed down the possibilities. For example, the size of the jitter was found to be independent of the amplitude of the orbits in the linac, so energy jitter coupled with linac dispersion is not the source, nor is fluctuations in quadrupole magnet (quad) currents. The possible contributions from quad vibrations led to measurements of the quad vertical motion [2]. Although the vibrations were found to be larger than expected, about $300 \mathrm{~nm}$ rms for some quads, simulations showed that even if all quads vibrated randomly by this amount, the resulting beam jitter could only account for a small fraction of the measured growth. Also, the frequency spectrum of the quad motion is highly peaked in the 8 to $14 \mathrm{~Hz}$ range which would not explain the relatively flat spectra of the beam jitter.

Computing the correlations of the jitter in each region of the linac with the jitter in other regions and with the jitter of other beam properties provided further clues. No strong correlation was found between the orbit jitter and the positron intensity, energy, or bunch length jitter. The correlations measured among the orbit data suggest that the growth is incoherent along the linac. Thus, the growth is not likely to be the result of large resonant amplification of betatron motion due to transverse wakefields. In fact, measurements of this amplification for oscillations induced at various points along the linac showed that it did not differ greatly from that expected, which is a factor of 2 to 3 at most.

One possible source that has not been ruled out is the transverse if fields in the S-band accelerator structures that result from dark currents. An rms kick of about $1 \mathrm{keV}$ per structure (12 m long) is needed to explain the observed level of jitter growth in the linac. Such a kick would be $2 \times 10^{5}$ times smaller than the accelerating field and would likely have a white noise spectrum. Another jitter source may be damping ring instabilities that produce $y-z$ correlations in the beam phase space that vary pulse-topulse. These correlations could vary in a manner that would not produce injection jitter but would nonetheless cause orbit changes in the linac due to the effect of the wakefields there. Further measurements will be made to assess the magnitude of these effects.

\section{SUMMARY}

Using a model-independent method, we have measured the positron vertical trajectory jitter in the SLC linac. We observe that the jitter grows three-fold by the end of the linac, increasing the time-averaged vertical beam emittance there by about $18 \%$. The trajectory jitter is essentially random pulse-to-pulse and is not strongly correlated with the positron intensity, energy, or bunch length jitter. Studies are continuing in an effort to determine the source of the jitter growth.

\section{REFERENCES}

[1] P. Raimondi, private communication (1995).

[2] J. Turner et. al., Vibration Studies of the Stanford Linear Accelerator, these proceedings (1995). 